\title{
LOS MODELOS DE USO TURÍSTICO DE LAS ÁREAS PROTEGIDAS DE CANARIAS: UNA PROPUESTA METODOLÓGICA
}

\author{
Moisés R. Simancas Cruz \\ Departamento de Geografía \\ Universidad de La Laguna
}

\section{RESUMEN}

Las categorías de protección ambiental determinan la adecuada gestión territorial de las áreas protegidas que comprenden y, por consiguiente, su correcta utilización como recurso turístico, en la medida en que fijan la relación de complementariedad -o, cuando menos, de neutralidad- entre esta funcionalidad y el fin último asignado a las mismas según las estrategias de manejo del riesgo de pérdida de su calidad ambiental derivado de la implementación de las actividades y equipamientos que requiere dicho uso turístico. Sin embargo, la elevada profusión, diversidad y heterogeneidad de las listas de denominaciones de las figuras ha provocado una elevada complejidad y confusión en su aplicación, dificultando, además, cualquier intento de estandarización y análisis comparado. Desde la vocación de ser un trabajo conceptual, instrumental y propositivo en materia de planificación turística del territorio, su principal objetivo es exponer una propuesta metodológica de optimización del uso turístico de las áreas protegidas basada en la definición de una serie de «modelos de gestión ambiental del territorio», que hemos denominado «de preservación», «de conservación» y mixto», y que, a modo de mecanismo clasificatorio de las categorías, permiten agruparlas en conjuntos homogéneos según sus semejanzas y regularidades en relación con las citadas estrategias.

Palabras clave: áreas protegidas, calidad ambiental, Canarias, capacidad de carga turística, categorías de protección ambiental, modelos de gestión ambiental del territorio, situación de riesgo, turismo.

\section{ABSTRACT}

The environmental protection categories determine the suitable management of the protected areas that they include and, consequently, your correct utilization as tourist resource, in the measurement in which they fix the complementarity relation —or, when less, neutrality relation - between this functionality and the last purpose assigned to the 
same ones according to the managing strategies the risk of loss of your environmental quality derived from the activities and equipments implementation that needs the above mentioned tourist use. Nevertheless, the high profusion, diversity and heterogeneity of the denominations list of such figures has motivated a high complexity and confusion in your application, impeding any attempt of standardization and compared analysis. From the vocation of being a conceptual, instrumental and propositive paper of for tourist planning of the territory, your principal aim is to expose a methodological offer of the tourist use optimization on the protected areas, based on the definition of «environmental management to the territory models», that we have named «preservation model», «conservation model» and «mixed model», like classification mechanism to the environmental protection categories, allow to group them in homogeneous sets according to your similarities and regularities in relation with the mentioned strategies.

Key words: protected areas, environmental quality, Canarias, tourist carrying capacity, management categories, environmental management models of the territory, risk situation, tourism.

\section{Introducción}

En consonancia con las distintas teorías de referencia utilizadas para discutir la evolución física y funcional de los espacios turísticos (Butler, 1980; Chadefaud, 1987; Miossec, 1977; Cadefaud, 1987; Cooper, 1994), diversos autores (Whelan, 1991; Machado, 1992; Organización Mundial del Turismo, 1993; UICN, 1994; Pedreño, 1996; Talaya, 1996) sostienen que mientras el modelo convencional de turismo de «sol y playa» se encuentra en una fase crítica de obsolescencia o de estancamiento, próximo al declive del ciclo de vida de los correspondientes destinos, otros productos se encuentran en plena madurez o desarrollo, como las denominadas genéricamente como «turismo rural», «turismo activo», «turismo en la naturaleza» o «turismo verde», para los que las áreas protegidas constituyen recursos turísticos adecuados y, por tanto, los escenarios territoriales óptimos de producción directa de bienes y servicios de consumo relativos a este tipo de ocio recreativo; en este sentido, éstas favorecen un punto de inflexión en el diseño de estrategias de cualificación, diversificación y diferenciación del turismo, así como una oportunidad y una ventaja competitiva en el marco de la globalización de los mercados turísticos. Como es sabido, esta dimensión turística se encuadra en el módulo temático de planificación y gestión territorial denominado de forma genérica como «uso público»y, aunque constituye una de las funcionalidades asignadas desde un principio a las mismas, resulta que ha adquirido una mayor relevancia, sobre todo, después del IV Congreso Mundial de Parques Nacionales y Áreas Protegidas de la UICN (Caracas, 1992) y de la publicación de «Parques para la Vida: Plan de Acción para las Áreas Protegidas de Europa» (1994) por la Comisión de Parques Nacionales y Áreas Protegidas y el Comité Europeo de Educación Ambiental de la UICN.

Aunque las áreas protegidas son consideradas como «bienes públicos» y «de interés general» por su mera existencia, así como por la valoración ética de la wildersness, con la consiguiente primacía de una concepción «ecocentrista», al fundamentarse tanto en posturas utópicas de apreciación de sus valores de uso no consuntivo —esto es, «de opción» y «de existencia»-, como en la generación de santuarios desde el predominio del idealismo platónico y de los planteamientos «antidesarrollistas», la posibilidad de ser objeto al mismo tiempo de cualquier tipo de utilización o aprovechamiento, actual o futuro (Pearce, 1993; Randall, 1987), como el turístico, permite su uso directo y, por tanto, el otorgamiento de 
una perspectiva utilitarista — instrumental—, en consonancia con la premisa de que «la protección medioambiental de un territorio en modo alguno puede ser sinónimo de abandono productivo o bloqueo de las actividades del mismo» (Baigorri, 1999). El reto consiste en compatibilizar este planteamiento «antropocéntrico» con el principal fin asignado a la política de declaración de las áreas protegidas, de manera que ésta no se conciba como un coste, sino como una ventaja competitiva, la cual se concreta, para este caso, en su capacidad, para asignar nuevas funciones territoriales relacionadas con el turismo a los ámbitos rurales, estructurar física y funcional de los espacios turísticos, generar una imagen de marca de un destino turístico, proceder a la tematización del producto turístico, reivindicando la «geografía de los lugares», así como diversificar y complementar a la modalidad turística de «sol y playa» (Simancas, 2004). Se trata, por tanto, del otorgamiento de un valor a las áreas protegidas en la medida de que satisfacen necesidades, en concreto, en forma de productos y servicios turísticos, circunstancia que, además de dotarlas de una dimensión estratégica, contribuye a justificar su razón de ser, así como a evitar que la política de protección ambiental del territorio sea una entelequia.

El uso turístico de las áreas protegidas de Canarias ha constituido una de las funciones vocacionales asignadas a las dos primeras declaraciones proteccionistas (1954) y posteriores (1974). Asimismo, aunque la intención de insertarlas en el proceso de turistización de la economía canaria - y, por ende, del territorio - no quedó reconocida formalmente ni en el texto legal que dio lugar a la «Red Canaria de Espacios Naturales Protegidos», la Ley 12/1987, de 19 de junio, de Declaración de Espacios Naturales Protegidos, ni en el que la completó conceptual y operativamente, la Ley 12/1994, de 19 de diciembre, de Espacios Naturales de Canarias, su diseño responde, entre otras cuestiones, a lograr «un desarrollo sostenido que tuviera en cuenta la estrecha relación que hay en Canarias entre el turismo - como base de la economía - y la oferta natural de las islas para atraer a dicho turismo» (Martín et al., 1995: 65). Esta pretensión ha sido asumida por las administraciones públicas canarias con competencias en materia turística y protección ambiental del territorio, de manera que en la Ley 7/1995, de 6 de abril, de Ordenación del Turismo en Canarias, éste se concibe como un sector que interactúa - positiva y negativamente- con «la protección del medio ambiente y conservación de la naturaleza, el paisaje y la cultura, en cuanto constituyen objetos de atracción y recursos turísticos» (artículo 1). Asimismo, en el Decreto Legislativo 1/2000, de 8 de mayo, por el que se aprueba el Texto Refundido de las Leyes de Ordenación del Territorio de Canarias y de Espacios Naturales de Canarias, que ha supuesto la unificación y adaptación de la planificación territorial de las áreas protegidas de la Ley 12/1994 a los nuevos criterios de integración propugnados en la Ley 9/1999, de 13 de mayo, de Ordenación del Territorio de Canarias, se fija que la ordenación de los recursos naturales se debe orientar a «la promoción de esos espacios [áreas protegidas] de la investigación científica, la educación ambiental y el encuentro del hombre con la naturaleza, en forma compatible con la preservación de sus valores» (artículo 2.2).

\section{Planteamiento del problema}

El uso turístico de las áreas protegidas requiere de dos componentes que, en gran medida, constituyen los mecanismos que posibilitan su transformación de «elementos patrimoniales» a «recursos turísticos» (Leno, 1993). Nos referimos, por una parte, a los equipamientos, entendidos como el conjunto de servicios, dotaciones, infraestructuras, instalaciones y medios — humanos y materiales — de distinta índole según su funcionalidad — de recepción y acogida de los usuarios, de divulgación, de recorrido, uso, conocimiento y disfrute del área protegida, así como de interpretación y educación ambiental—, y por otra, a la oferta 
ampliamente diversificada de actividades, que conforman los programas de acogida de sus usuarios. Ambos facilitan, e incluso, favorecen, el uso y disfrute turístico - y, por extensión, público- de las áreas protegidas (Corraliza, García y Valero, 1999), de forma individual o colectiva, espontánea u organizada (Organismo Autónomo Parques Nacionales, 2000), por parte de un turista o un visitante - excursionista - según la distinción realizada por la Organización Mundial del Turismo. Asimismo, contribuyen a que sea más conveniente el uso de la expresión «turismo de áreas protegidas» en detrimento de «turismo en áreas protegidas», por cuanto se trata de que éstas no sean utilizadas como meros soportes físicos sino como «recursos focales»y, por consiguiente, como los factores centrales, estructurantes y distintivos de atracción del flujo turístico; de esta manera, tanto los equipamientos como la propia práctica de las actividades turísticas constituyen los atractivos «complementarios» y «de apoyo», de modo que, si bien se comportan como los elementos que por sí solos no constituyen el motivo o factor suficiente de persuasión para la visita, contribuyen, entre otras cuestiones, al incremento del valor de las áreas protegidas como recurso de ocio, así como del grado de satisfacción y diversidad de la experiencia recreativa tanto a escala local —en la propia área protegida— como global — del destino turístico—.

Resulta, por tanto, que el uso turístico de las áreas protegidas precisa el acondicionamiento de las mismas, con el fin de adaptarlo y adecuarlo a las exigencias y necesidades de su usuario. Esta circunstancia induce a que este tipo de uso es susceptible de generar impactos ambientales de signo negativo, en la medida en que modifica el sistema territorial objeto de protección ambiental; en este sentido, la turistización de las áreas protegidas puede comportarse de una forma muy similar al de las áreas turísticas del litoral, con la única diferencia que las perturbaciones negativas se producen a un ritmo más lento (Wearing y Neil, 2000). Asimismo, es frecuente que la afluencia «de masas», masiva e incontrolada de los visitantes/turistas se centre en determinados hitos del área protegida, con la consiguiente concentración espacial y temporal, así como un uso excesivo, intensivo y no adecuado de los equipamientos disponibles, induciendo a la congestión y saturación turística, que, en algunas ocasiones, llegan a un grado realmente crítico en consonancia con el modelo del ciclo de vida en los destinos de naturaleza planteado por Vera et al. (1997), incidiendo, incluso, sobre la propia experiencia recreativa, al reducir o atenuar su calidad; a este respecto, resulta que normalmente tales efectos adversos se asemejan al fenómeno de «tiranía de las pequeñas decisiones» observado para otros supuestos por Kahn (1971), en la medida en que el deterioro del ámbito territorial deriva de la acumulación de un elevado número de actuaciones aisladas, que, aunque ninguna en particular tiene consecuencias desfavorables, en su conjunto genera un impacto agregado y sinérgico, cuya gravedad se incrementa de manera progresiva al prolongarse la acción que lo genera, así como por el reforzamiento de los efectos simples. El resultado es que es frecuente que el uso turístico de las áreas protegidas se proyecte como una «amenaza», en cuanto genera situaciones de conflictividad, incompatibilidad, disfuncionalidad, colisión o tensión que dificultan el logro del objetivo primordial de su declaración como tales, esto es: lograr indefinidamente la permanencia de la integridad de la calidad ambiental de las características o cualidades intrínsecas de sus contenidos geográficos y, por tanto, de los elementos constituyentes —abióticos, bióticos y antrópicos- de los sistemas territoriales que van a configurar el «hecho protegido», así como de los procesos que los relacionan, entendida como el mérito, valor o grado de excelencia para que no se destruyan o deterioren (Conesa, 1997), favoreciendo, incluso, su mejora mediante acciones de restauración y/o recuperación (Simancas, 2003).

La única opción de romper este ciclo es intervenir sobre el propio desarrollo territorial del turismo, así como en la forma de su aprovechamiento y utilización turística mediante la selección de la opción más adecuada, es decir, aquella que maximice la aptitud del territorio 
para acoger dicho uso, minimizando los impactos negativos. La declaración de áreas protegidas permite lograr este principio, en la medida en que, como un producto territorial formal y concreto derivado de la materialización espacial de la política de protección ambiental del territorio, supone una actuación pública que, en esencia, implica la identificación, selección, valoración y delimitación geográfica de un conjunto de unidades territoriales —las áreas protegidas - en función de una serie de criterios, atributos — cuantitativos y cualitativos- y cualidades — los denominados fundamentos de protección ambiental-, cuya agrupación configura una totalidad o globalidad — la red de áreas protegidas - que, a vez, se clasifica en tipos - las categorías o figuras de protección ambiental del territorio-, a las que se otorga un régimen legislativo específico, de manera que quedan singularizadas respecto al contexto geográfico donde se insertan, siendo objeto de un tratamiento específico y homogéneo por parte de los correspondientes instrumentos de planificación ambiental — planes y normas de conservación - , a los que queda sujeta su gestión territorial. Por consiguiente, las áreas protegidas son el resultado de la implementación de una estrategia de gobierno del territorio dirigida a la transformación de las situaciones preoperacionales — con anterioridad a su declaración como «protegidas»— de incertidumbre, en la que ni se conocen ni se pueden controlar las posibilidades de que el uso turístico exceda un valor específico de consecuencias - impactos - no deseadas que afecten negativamente a la calidad ambiental de las áreas protegidas, en otras de riesgo, en las que se conocen tanto las probabilidades de ocurrencia efectiva de tales efectos adversos como su intensidad o magnitud, entendida como la medida cuantitativa o cualitativa de su severidad, convirtiéndolos, de este modo, en esperados y, por tanto, en evitables a través de la ordenación física y funcional del sistema territorial objeto de protección ambiental. Desde esta perspectiva, las categorías van a constituir las referencias que guían el manejo adecuado de dicha situación de riesgo y, por tanto, los esquemas de referencia del proceso de optimización territorial del uso turístico de las áreas protegidas que comprenden. Así, los diferentes tipos y formas de uso turístico de las áreas protegidas deben ajustarse a la imagen de un futuro posible y deseable proyectado de manera implícita en cada figura legal de protección ambiental, el cual supone un escenario normativo - y, por tanto, de anticipación (Lleó de la Viña, 1979) — relativo a los objetivos concretos a alcanzar a este respecto, y que quedan definidos de manera prospectiva para cada una en el momento de su formalización y aprobación legislativa.

Sin embargo, la elevada profusión, diversidad y heterogeneidad a escala mundial -e incluso, nacional — de la lista de denominaciones y categorías - y, por tanto, de tipos de áreas protegidas - puesta de manifiesto, entre otros, por Azcárate y Aboal (1996), Fernández, Pérez y de Lucio (1997), Mulero (2002) y Troitiño y otros (2005), la limitación a meras nomenclaturas de las sucesivas iniciativas internacionales de homogenización — por ejemplo, las del Comité de Ministros del Consejo de Europa (1973), FAO/PNUD (1975), UICN (1969; 1972; 1978; 1994)—, así como la utilización de diferentes criterios en la definición de las directrices, funcionalidades y objetivos concretos de gestión territorial, constituyen algunos factores que han provocado una elevada complejidad y confusión en su aplicación, a la vez que dificultan cualquier intento de estandarización y análisis comparado. Esta situación es la que caracteriza a la política española de declaración de áreas protegidas, en la medida en que la capacidad constitucional otorgada a las Comunidades Autónomas de promulgar sus propias normas en esta materia ha provocado una importante inflación y diversificación de las cinco categorías - parques, parques nacionales, reservas naturales, monumentos naturales y paisajes protegidos - contempladas en la Ley 4/1989, de 27 de marzo, de Conservación de los Espacios Naturales y de la Flora y Fauna Silvestre, generándose un total de treinta y cinco figuras diferentes, siendo el 77,1\% de aplicación exclusiva en el ámbito geográfico de la norma jurídica autonómica que la ha originado (cuadro 1). 
Cuadro 1

ANÁLISIS COMPARADO DE LA DISTRIBUCIÓN DEL USO DE LAS CATEGORÍAS DE PROTECCIÓN AMBIENTAL DEL TERRITORIO SEGÚN LAS COMUNIDADES AUTÓNOMAS ESPAÑOLAS

\begin{tabular}{|c|c|c|c|c|c|c|c|c|c|c|c|c|c|c|c|c|c|c|}
\hline \multicolumn{2}{|c|}{$\begin{array}{l}\text { Categorías de protección } \\
\text { ambiental del territorio }\end{array}$} & 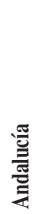 & 㤐 & 尊 & 离 & 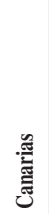 & 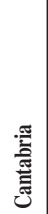 & 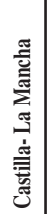 & 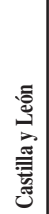 & 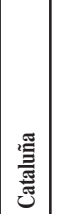 & 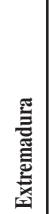 & : & 를 & 竞 & 焉 & 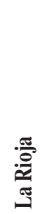 & 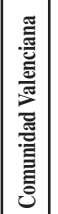 & 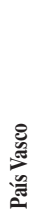 \\
\hline \multicolumn{19}{|c|}{ Árbol Singular } \\
\hline \multicolumn{19}{|c|}{ Área Natural Recreativa } \\
\hline \multicolumn{19}{|c|}{ Biotopo Protegido } \\
\hline \multicolumn{19}{|c|}{ Corredor Ecológico y de Biodiversidad } \\
\hline \multicolumn{19}{|c|}{ Enclave Natural } \\
\hline \multicolumn{19}{|c|}{$\begin{array}{l}\text { Espacio Natural en Régimen de Protección } \\
\text { General }\end{array}$} \\
\hline \multicolumn{19}{|c|}{ Espacio Natural Protegido } \\
\hline \multicolumn{19}{|c|}{ Microrreserva } \\
\hline \multirow{2}{*}{\begin{tabular}{l|l} 
Monumento \\
Natural
\end{tabular}} & Monumento Natural & & & & & & & & & & & & & & & & & \\
\hline & de Interés Nacional & & & & & & & & & & & & & & & & & \\
\hline \multicolumn{19}{|c|}{ Paisaje Protegido } \\
\hline \multirow{4}{*}{ Paraje } & Natural & & & & & & & & & & & & & & & & & \\
\hline & Natural de Interés Nacional & & & & & & & & & & & & & & & & & \\
\hline & Natural Municipal & & & & & & & & & & & & & & & & & \\
\hline & Pintoresco & & & & & & & & & & & & & & & & & \\
\hline \\
\hline \multicolumn{19}{|c|}{$\begin{array}{l}\text { Parque Nacional } \\
\\
\end{array}$} \\
\hline \multirow{4}{*}{ Parque } & Periurbano & & & & & & & & & & & & & & & & & \\
\hline & $\begin{array}{l}\text { Periurbano de Conservación } \\
\text { y Ocio }\end{array}$ & & & & & & & & & & & & & & & & & \\
\hline & Regional & & & & & & & & & & & & & & & & & \\
\hline & Rural & & & & & & & & & & & & & & & & & \\
\hline \multicolumn{19}{|c|}{ PEIN (Plan de Espacios de Interés Natural) } \\
\hline \multicolumn{19}{|c|}{ Plan Especial de Protección } \\
\hline \multirow{8}{*}{$\begin{array}{l}\text { Reserva } \\
\text { Natural }\end{array}$} & Reserva Natural & & & & & & & & & & & & & & & & & \\
\hline & Concertada & & & & & & & & & & & & & & & & & \\
\hline & de Fauna Salvaje & & & & & & & & & & & & & & & & & \\
\hline & Dirigida & & & & & & & & & & & & & & & & & \\
\hline & Especial & & & & & & & & & & & & & & & & & \\
\hline & Integral & & & & & & & & & & & & & & & & & \\
\hline & Marina & & & & & & & & & & & & & & & & & \\
\hline & Parcial & & & & & & & & & & & & & & & & & \\
\hline \multicolumn{19}{|c|}{ Reserva (ley propia) } \\
\hline Reserva $\mathbf{F}$ & luvial & & & & & & & & & & & & & & & & & \\
\hline Reserva I & tegral & & & & & & & & & & & & & & & & & \\
\hline Refugio d & Fauna & & & & & & & & & & & & & & & & & \\
\hline Régimen & Protección Preventiva & & & & & & & & & & & & & & & & & \\
\hline Reserva d & e la Biosfera & & & & & & & & & & & & & & & & & \\
\hline Sitio de In & terés Científico & & & & & & & & & & & & & & & & & \\
\hline Sitio Natu & ral de Interés Nacional & & & & & & & & & & & & & & & & & \\
\hline Zona Esp & cial de Conservación & & & & & & & & & & & & & & & & & \\
\hline
\end{tabular}

Fuente: EUROPARC-España (2004). Elaboración propia. 
Este problema plantea la necesidad de proponer una clasificación de las categorías de protección ambiental en grupos homogéneos que, superando los esquemas basados en simples listas de nombres, posibilite la homologación de los patrones de gestión territorial a diferentes ámbitos escalares, la simplificación de su estudio comparativo, así como la identificación y estructuración de las semejanzas, regularidades o diferencias entre los mencionados objetos y, en este caso, en las formas de intervención territorial sobre las situaciones de riesgo de perjuicio de la calidad ambiental de las áreas protegidas que implica su uso turístico. Partimos de la premisa - a demostrar - que es su división mediante la definición de lo que hemos denominado «modelos de gestión ambiental del territorio», permite la sistematización de las figuras legales de protección ambiental y, por tanto, su ordenación o agrupación, según la función de pertenencia u homogeneidad en relación con las estrategias de manejo de las citadas situaciones de riesgo.

Así, desde la intención de ser un trabajo conceptual, instrumental y propositivo en materia de planificación y gestión turística del territorio, su principal objetivo es exponer el procedimiento metodológico seguido en el consiguiente proceso de modelización. Si bien se trata de una de tantas propuestas de clasificación funcional de las categorías de protección que se pueden realizar en función de los distintos enfoques que pueden darse y que, en este caso, se verá corroborada por la aplicación que pretendemos darle, esto es, la optimización para la mejor utilización turística de las áreas protegidas. En este sentido, pretendemos contribuir modestamente a romper con la tendencia diagnosticada en el Plan de Acción para los Espacios Naturales Protegidos del Estado español (EUROPARC-España, 2002) de que la investigación no se ha consolidado aún como un pilar imprescindible para la planificación y gestión de las áreas protegidas. De esta manera, se trata de un trabajo que pretende insertarse en las iniciativas de convertirlas en laboratorios de investigación aplicada, así como contribuir a las acciones prioritarias propuestas en dicho Plan dirigidas a la aportación de protocolos y metodologías rigurosas, estandarizadas y comparables relativas a ambas labores técnicas, la necesaria difusión de los resultados de las investigaciones en esta materia, así como el desarrollo de las correspondencias entre las categorías de protección ambiental locales —nacionales y autonómicas- y las de la UICN.

Con el fin de aportar algo de contenido empírico — no especulativo- y dotar de una dimensión aplicada a los diferentes enunciados generales y al planteamiento metodológico que se va a exponer, nos basamos en la evidencia paradigmática de Canarias. Su estructura territorial, configurada por 146 áreas protegidas que ocupan 309.755,9 hectáreas, hace que resulte un adecuado laboratorio espacial en materia de protección ambiental del territorio, en la medida en que, derivado a su elevado porcentaje de superficie protegida en relación a la total (el 41,6\%), encabeza el grupo de las Comunidades Autónomas españolas que superan la superficie media nacional $(10,6 \%)$, así como ocupa la segunda posición en cuanto al número de áreas protegidas y la cuarta en lo relativo a la superficie protegida en términos absolutos. Asimismo, la discontinuidad geográfica inherente a su condición archipielágica - el denominado «hecho insular»-, incrementa, si cabe, el interés de la investigación, debido al impacto muy significativo de las áreas protegidas sobre la organización territorial de cada isla: considerando que la media insular es del 41,8\%, resulta que, a excepción de Fuerteventura, el porcentaje de superficie protegida supera el 30\% de la extensión total, llegando a sobrepasar el $40 \%$ en el caso de Lanzarote y Gran Canaria, e incluso, el 50\% en los de Tenerife y El Hierro. 


\section{Material y método. Los criterios de clasificación de las estrategias de optimización territorial del uso turístico de las áreas protegidas en función de las situaciones de riesgo de pérdida de su calidad ambiental}

En consonancia con las conclusiones del Taller 4, Gestión del Uso Público, del $7^{\circ}$ Congreso de EUROPARC-España (2001), la funcionalidad recreativa — real o potencial- de las áreas protegidas se fundamenta en la implementación de un modelo de uso turístico que posibilite la vertebración adecuada de la relación entre el medio protegido y las actividades y equipamientos que requiere. Considerando el objetivo planteado en este trabajo, a continuación se definen los criterios y factores base que definen o intervienen en el procedimiento de formulación de los modelos de gestión ambiental del territorio y, por tanto, en el proceso metodológico de obtención de la asignación espacial del uso turístico que se puede desarrollar en un área protegida de mayor probabilidad de ser la óptima.

\subsection{Los factores de asignación del uso turístico más adecuado}

El proceso de modelización se ha iniciado con la identificación de los elementos que intervienen en la caracterización de cualquier mecanismo de asignación óptima de usos a las áreas protegidas. De esta manera, ha supuesto la convergencia en justo equilibrio, así como la valoración cualitativa mediante operaciones empíricas y sistémicas (Antón y Vera, 1998), de los dos sentidos interrelacionados de la citada relación, dando lugar al binomio «impacto-aptitud» (Gómez Orea, 1992) que resulta de la consideración de sendas perspectivas: la del medio y la de la propia actividad turística.

Desde el punto de vista de la primera, se trata de considerar la fragilidad o vulnerabilidad de los contenidos geográficos de las áreas protegidas, entendida como su incapacidad para absorber los efectos del cambio que genera el proceso de turistización y, por ende, de adaptación al mismo, con el menoscabo de las cualidades o méritos que justificaron su declaración como tales, así como la consecuente reducción del valor de protección. Se trata, por tanto, del factor interno del sistema territorial objeto de protección ambiental, de manera que su diferencia determina el carácter selectivo de la severidad de los efectos de las actividades y equipamientos turísticos en función de la premisa de que «a una misma intensidad de actuación [turística], se causará un impacto negativo de mayor gravedad allí donde la fragilidad sea mayor» (Ramos et al, 1995: 287). Su determinación implica tener en cuenta la predisposición intrínseca de un elemento o grupo de elementos a ser afectado o de ser susceptible a padecer una pérdida de su calidad ambiental ante la ocurrencia efectiva de las consecuencias o alteraciones intensas adversas derivadas de su uso turístico sobre los fundamentos de protección ambiental del territorio y, por consiguiente, el conjunto de atributos y cualidades de estimación cuantitativa y cualitativa que le han otorgado el mérito para ostentar tal condición. Por tanto, considerando que la vulnerabilidad deriva de la agregación de los fundamentos de protección ambiental (figura 1), resulta que éstos no sólo van a constituir los recursos focales de las áreas protegidas y, por tanto, los factores de atracción turística más importantes (Simancas, 2004), sino que también van a condicionar su desarrollo óptimo, en la medida en que su mantenimiento constituye en sí mismo un objetivo preciso de su gestión territorial.

Por su parte, desde la perspectiva del medio, el proceso de optimización precisa de la consideración de la potencialidad o aptitud de las áreas protegidas para acoger uso turístico, la cual hace referencia a las propiedades o características que determinan su capacidad o predisposición intrínseca para proveer un conjunto de posibilidades y condiciones óptimas para dar cabida a las actividades y equipamientos que requiere sin que se produzca una 


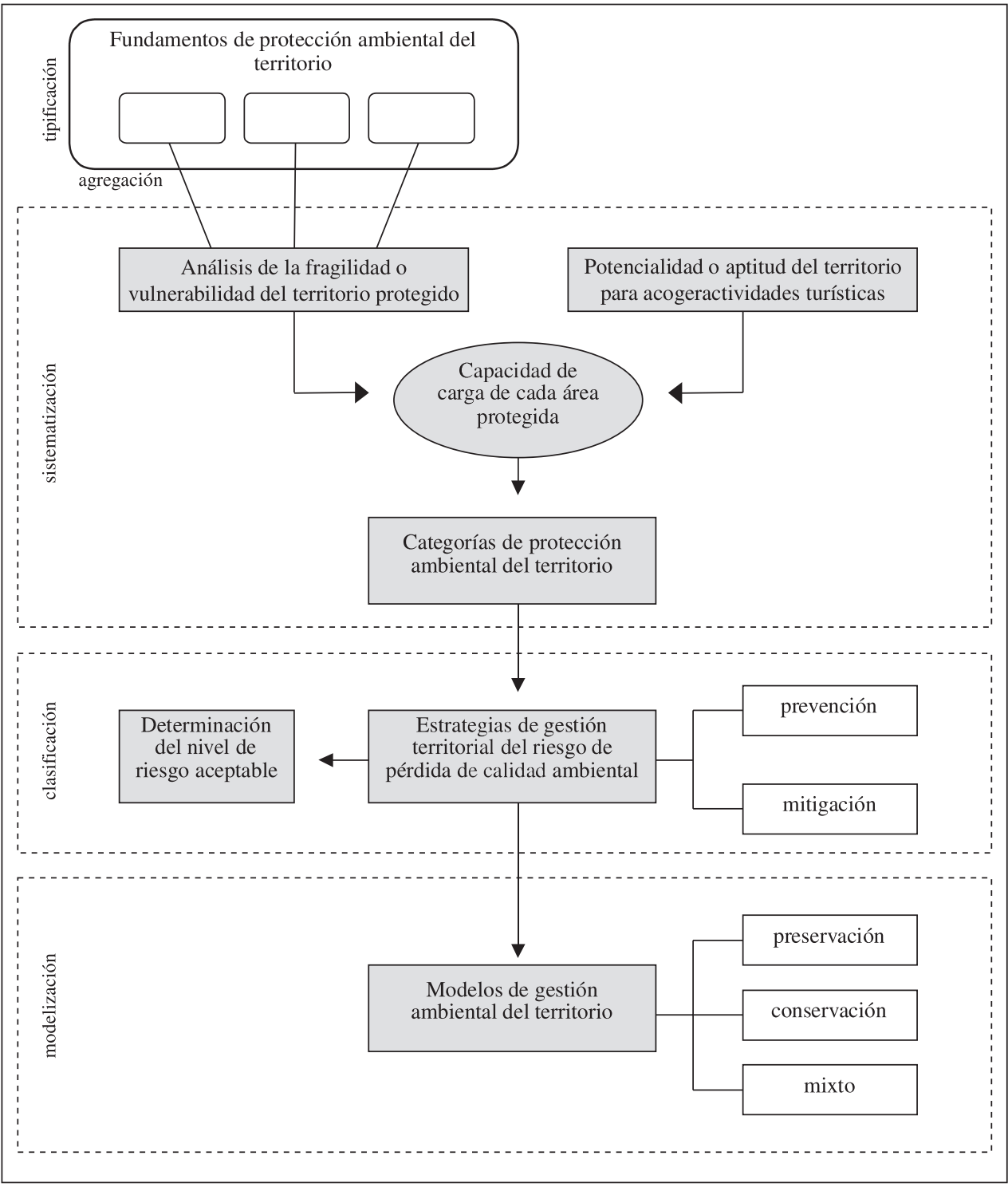

Figura 1. Proceso metodológico de definición de los modelos territoriales de gestión ambiental de las áreas protegidas.

pérdida de su calidad ambiental. Su consideración supone un reconocimiento implícito de que el desarrollo óptimo del uso turístico no sólo depende de las condiciones físicas - territoriales - de las características y elementos significativos de las áreas protegidas, sino también de las necesidades espaciales de las modalidades que se pretenden acoger, así como de los componentes psicológicos de los usuarios, que hacen alusión a su percepción subjetiva en términos del grado de satisfacción en función de sus expectativas, experiencias 
previas, etc. Así, cada modalidad turística presenta óptimos, tanto en lo material, esto es, en el requerimiento de equipamientos físicos para acogerlo, como en lo psicológico, entendido como la percepción subjetiva de los usuarios en términos del grado de satisfacción, los cuales lógicamente para cada una de las actividades y sus usuarios. En consecuencia, cuando los factores y elementos que posibilitan la capacidad recreativa de las áreas protegidas son propicios y concurrentes sobre tales óptimos, éstos constituyen los valores que determinan su desarrollo adecuado. De esta manera, la determinación del potencial del medio implica la identificación de la tipología e intensidad del uso turístico más viable, en la medida en que no afecte negativamente tanto a la propia experiencia recreativa como al ámbito geográfico; para ello resultan esenciales las condiciones en que se desarrolla en términos de calidad de recurso, grado de saturación, el tipo de práctica recreativa esperada, la incompatibilidad entre practicantes de actividades diversas, etc. (Viñals et al., 2002). El resultado es que, mientras un área protegida puede cubrir los requisitos de localización de una determinada actividad turística, su aptitud es baja para otro tipo, circunstancia que genera una afección negativa a su desarrollo óptimo, surgiendo, incluso, conflictos entre distintos usuarios y entre éstos y los técnicos-gestores de las mismas. Además, la limitación del número de encuentros o experiencias no deseadas en lo relativo a tales actividades, restringiendo la aparición de conductas inadecuadas y reduciendo los conflictos entre usuarios, puede aumentar la aptitud de un área para acogerlas; así, por ejemplo, mientras que la capacidad perceptual de un birdwatchers es muy baja, debido a su exclusividad y la reducida o nula tolerancia de la presencia de demasiados usuarios, ni aún desarrollando la misma actividad, la de los grupos de escolares que desarrollan actividades educativas es alta, pudiendo constituir grupos más numerosos, llegando, incluso, a soportar la interacción con otras modalidades lúdicas (Viñals et al, 2002).

A efectos de estas consideraciones, las áreas protegidas presentan una elevada diversidad en cuanto al binomio impacto-aptitud y, por consiguiente, a las posibilidades que pueden soportar un uso turístico acorde a sus particularidades y a las formas de su desarrollo conveniente, en cuanto permite fijar su compatibilidad con el mantenimiento de los fundamentos de protección ambiental del territorio. La concurrencia e integración en conformidad de ambos elementos permite agruparlas en función de las semejanzas y regularidades en cuanto a su «capacidad de carga» —-física, material y psicológica o perceptual (O`Reilly, 1986) — para acogerlas (figura 1). Aunque su determinación se fundamenta en modelos teóricos de difícil aplicación y concreción objetiva, su utilidad práctica y extensa la hace adecuada como una herramienta y técnica para sustentar las decisiones técnicas de planificación y gestión territorial de las áreas protegidas y, por ende, de su ordenación física y funcional; en este sentido, no debemos obviar que, aunque los primeros antecedentes de su uso se remontan a la década de los treinta, cuando las ciencias biológicas comenzó a utilizarlo para determinar el número de animales de una especie concreta que un hábitat específico puede soportar (Dasmann, 1964), fue en la de los sesenta y setenta cuando adquirió una cierta importancia, coincidiendo con la consolidación de la funcionalidad turística de las áreas protegidas y, sobre todo, con la idea de encontrar y aplicar técnicas destinadas a establecer los tipos, ritmos y niveles de uso más apropiados. La capacidad de carga turística de cada área protegida constituye la expresión, a modo de indicador, del «grado de idoneidad o cabida [...], teniendo en cuenta a la vez, la medida en que el medio cubre sus requisitos locacionales y los efectos de dicha actividad [y los equipamientos] sobre el medio (Gómez, 1992: 25). Considerando que su determinación se realiza en función tanto de los factores de lectura objetiva de las características de sus equipamientos y actividades — tipología de la demanda, frecuentación de las visitas, etc.—, como de la calidad ambiental de las áreas protegidas, su aplicación al proceso de adecuación territorial del uso turístico de las áreas 
protegidas permite la selección de la mejor opción, mediante su asignación, distribución y regulación en términos de neutralidad, compatibilidad, coexistencia, indeferencia o armonización y, en el mejor de los casos, de complementariedad, simbiosis, e incluso, sinergia - reforzamiento - respecto a la calidad ambiental de las mismas (cuadro 2). Desde este punto de vista, la práctica del turismo en las áreas protegidas pueden beneficiarse de una serie de fortalezas que la distingue del modo en que se produce en los ámbitos territoriales no protegidos, en consonancia con el principio de sostenibilidad en materia turística expresado por la Organización Mundial del Turismo y el Programa de las Naciones Unidas sobre Medio Ambiente (1992): «El turismo sostenible sólo puede tener lugar si se establecen capacidades de carga para los destinos turísticos clave y después se respetan esas capacidades de forma rigurosa mediante un sistema de planificación eficaz y llevando a cabo revisiones del funcionamiento. Estos estudios y normativas constituirán la piedra angular de los planes y estrategias turísticas locales a largo plazo [...]».

\subsection{La definición del criterio de clasificación}

La clasificación de las diversas capacidades de carga turística de las áreas protegidas permite su agrupación en conjuntos homogéneos, las categorías o figuras de protección ambiental del territorio, en función de las semejanzas relativas al riesgo inherente a su uso turístico de pérdida o daño - por deterioro o destrucción — de la calidad ambiental de las áreas protegidas que comprenden. Así, cada categoría expresa un valor que refleja la capacidad de acogida de las áreas protegidas que comprende, el cual deriva de la identificación, análisis y discriminación en términos cualitativos de la probabilidad de que, debido a un uso turístico inadecuado, se presenten ciertas consecuencias no deseadas al exceder un determinado nivel de severidad, afectando negativamente a los fundamentos de protección ambiental expuestos, con la consiguiente pérdida de calidad ambiental de las áreas protegidas, así como obstaculizando el cumplimiento de los objetivos concretos de gestión territorial asignados.

Desde el punto de vista metodológico, la determinación del riesgo deriva de la convergencia de dos factores básicos en interacción — los cambios en uno o en los dos modifican el riesgo- : las amenazas y la vulnerabilidad de los contenidos geográficos objeto de protección ambiental. Las primeras constituyen las acciones intraespaciales —origen interno al área protegida-, intraterritorial - interno al contexto territorial donde se inserta- e interterritorial —externo a dicho sistema territorial—, que, a modo de agentes «perturbadores», derivan de las características, dimensiones y ubicaciones geográficas de los equipamientos y del propio desarrollo de las actividades turísticas; su evaluación supone la estimación tanto de la probabilidad de que se manifieste su impacto con potencial de alteración de la calidad ambiental de las áreas protegidas, como de su severidad, la cual es inversamente proporcional a la probabilidad de la materialización efectiva de las amenazas. Por su parte, la vulnerabilidad está íntimamente relacionada no sólo con su mencionada predisposición intrínseca sino también con el grado esperado o susceptible de padecer daño y, por tanto, a padecer la pérdida de calidad ambiental, constituyendo, por tanto, el factor de riesgo interno que incide sobre la capacidad de que la amenaza genere una afección, siendo directamente proporcional a su severidad; desde esta perspectiva, la vulnerabilidad lleva implícita el grado de «exposición», que hace referencia a los «elementos y procesos objeto de protección en riesgo»y, por consiguiente, los fundamentos de protección ambiental que pueden verse afectados por la ocurrencia y manifestación efectiva de las amenazas. Esta relación sistémica es susceptible de ser representada matemáticamente - $-\mathrm{y}$, por tanto, de que la evaluación del riesgo adquiera una dimensión cuantitativa - según la siguiente formulación general, 
ampliamente aceptada y aplicada en los trabajos de Análisis de Riesgos (UNDRO, 1979; Olcina y Ayala-Carcedo, 2002):

$\begin{array}{llll}\text { donde: } & \mathrm{R}= & \text { el riesgo } & \mathrm{R}=\mathrm{A} \times \mathrm{V} \\ \mathrm{A} & = & \text { las amenazas } & \\ \mathrm{V}= & \text { la vulnerabilidad o fragilidad }\end{array}$

En consecuencia, la política de declaración de áreas protegidas en general, y las iniciativas de adecuación territorial de su uso turístico en particular, supone un mecanismo de gestión del citado riesgo, en la medida en que permite la disminución de la propensión, susceptibilidad o tendencia de la totalidad o parte del área protegida a ser afectada por la ocurrencia efectiva de sus consecuencias. Así, desde el punto de vista operacional, el proceso de optimización territorial del uso turístico de las áreas protegidas supone una estrategia eficaz de reducción del riesgo, al dirigir la acción - y, por consiguiente, intervenir - sobre alguno de los factores de dicha operación, con el fin de que tienda a una intensidad igual a cero, bien las amenazas, con lo que $\mathrm{R}=0 \mathrm{x} \mathrm{V}$, bien la vulnerabilidad, de modo que $\mathrm{R}=\mathrm{A}$ x 0 . De este modo, considerando los extremos de la escala de medición de dicho riesgo — de alta probabilidad pero baja magnitud y de baja probabilidad pero gran magnitud-, se logra que el resultado disminuya proporcionalmente, reduciendo el riesgo al mínimo hasta aproximarse, incluso, a cero ( $\mathrm{R}$ $=0$ ), aunque los fundamentos de protección ambiental continúen estando expuestos al mismo. Así, aunque las áreas protegidas que comprenden las categorías de protección ambiental de la Red Canaria presentan diferentes grados de vulnerabilidad (cuadro 3), las estrategias de optimización - y, por ende, de gestión del riesgo de daño de su calidad ambiental- pueden clasificarse en dos tipos según la acción se dirija hacia uno u otro de los elementos que lo configuran (cuadro 2):

a) De prevención. Consiste en una estrategia proteccionista fundamentada en la anulación de cualquier factor externo de riesgo de alteración negativa —actual o potencial- y, por tanto, la intervención directa sobre la ocurrencia efectiva de las amenazas. Se trata de «retirar» los elementos objeto de protección ambiental expuestos a las mismas, mediante la prohibición total de cualquier tipo de actividad y equipamiento de uso turístico, a excepción de aquellos con fines científicos, educativos o técnicos, previa autorización administrativa, impidiendo o evitando así la ocurrencia de las acciones que pueden dañar su calidad ambiental.

b) De mitigación. Supone incidir sobre el otro factor de la relación con el fin de proporcionar mayores niveles de seguridad — o menor vulnerabilidad — , ya que las medidas anticipadas que conlleva esta estrategia de gestión permiten la disminución, control o supresión de la fragilidad de los elementos y procesos bajo riesgo - y, por ende, del grado de exposición-, al incidir sobre su impotencia para absorber las modificaciones introducidas por las actividades y los equipamientos inherentes al proceso de turistización de las áreas protegidas, atenuando significativamente las consecuencias esperadas y potenciales. Así, el proceso de optimización del uso turístico de las áreas protegidas supone su adecuación a su «resiliencia», que, entendida como el anverso de la vulnerabilidad, implica la consideración de su capacidad o grado de adaptación para mantener o recuperar rápidamente su calidad ambiental por la materialización efectiva de un impacto perjudicial; así, las áreas protegidas de mayor resiliencia son las que registran puntuaciones bajas de vulnerabilidad. 
Cuadro 2

CARACTERÍSTICAS DE LOS MODELOS DE GESTIÓN AMBIENTAL DEL TERRITORIO EN RELACIÓN CON EL USO TURÍSTICO DE LAS ÁREAS PROTEGIDAS

\begin{tabular}{|c|c|c|c|c|c|}
\hline & & & PRESERVACIÓN & CONSERVACIÓN & MIXTO \\
\hline \multicolumn{3}{|c|}{$\begin{array}{l}\text { TIPO DE ESTRATEGIA DE GESTIÓN TERRITORIAL DE } \\
\text { LA SITUACIÓN DE RIESGO }\end{array}$} & De prevención & \multicolumn{2}{|c|}{ De mitigación } \\
\hline \multirow{2}{*}{$\begin{array}{c}\text { DOMINANCIA } \\
\text { DE } \\
\text { ELEMENTOS }\end{array}$} & \multicolumn{2}{|c|}{ Grado de naturalidad } & 4 & 1 & 2 \\
\hline & \multicolumn{2}{|c|}{ Grado de antropización } & 0 & 3 & 2 \\
\hline \multirow{9}{*}{$\begin{array}{l}\text { OBJETIVOS Y } \\
\text { PRORIDADES } \\
\text { DE LA } \\
\text { GESTIÓN } \\
\text { TERRITORIAL* }\end{array}$} & \multicolumn{2}{|c|}{ Investigación científica } & A & $\mathrm{C}$ & B \\
\hline & \multicolumn{2}{|c|}{ Educación e interpretación ambiental } & - & B & B \\
\hline & \multicolumn{2}{|c|}{ Turismo y recreación } & - & B & A \\
\hline & \multicolumn{2}{|c|}{$\begin{array}{l}\text { Mejora de la calidad de vida de las comunidades } \\
\text { locales }\end{array}$} & - & A & - \\
\hline & \multicolumn{2}{|c|}{ Utilización sostenible de los recursos } & - & A & $\mathrm{C}$ \\
\hline & \multicolumn{2}{|c|}{$\begin{array}{l}\text { Protección de los sistemas naturales y de los } \\
\text { hábitats característicos }\end{array}$} & A & B & A \\
\hline & \multicolumn{2}{|c|}{$\begin{array}{l}\text { Protección de especies y la diversidad genética } \\
\text { (biodiversidad) }\end{array}$} & A & A & A \\
\hline & \multicolumn{2}{|c|}{ Protección de los procesos ecológicos esenciales } & A & A & A \\
\hline & \multicolumn{2}{|c|}{$\begin{array}{l}\text { Protección de los atributos culturales, } \\
\text { tradicionales y paisajísticos }\end{array}$} & - & A & B \\
\hline \multirow{4}{*}{$\begin{array}{l}\text { CAPACIDAD } \\
\text { DE CARGA } \\
\text { PARA } \\
\text { ACOGER USO } \\
\text { TURÍSTICO }\end{array}$} & \multicolumn{2}{|c|}{ Potencialidad } & 0 & 3 & 2 \\
\hline & \multicolumn{2}{|c|}{ Fragilidad } & 4 & 1 & 2 \\
\hline & \multicolumn{2}{|c|}{ Tipo de relación «actividad turística - medio» } & Conflictividad & $\begin{array}{c}\text { Neutralidad / } \\
\text { complementariedad }\end{array}$ & $\begin{array}{l}\text { Complementa- } \\
\text { riedad }\end{array}$ \\
\hline & \multicolumn{2}{|c|}{$\begin{array}{l}\text { Capacidad de carga del área protegida para } \\
\text { acoger actividades turísticas }\end{array}$} & $0-1$ & $3-4$ & $2-3$ \\
\hline \multicolumn{3}{|c|}{ TIPO DE GESTIÓN TÉCNICA } & Activa & \multicolumn{2}{|c|}{ Pasiva - Intermedia } \\
\hline \multicolumn{2}{|c|}{$\begin{array}{l}0-\text { Nula } \\
1-\text { Baja } \\
2-\text { Media } \\
3-\text { Alta } \\
4-\text { Muy alta }\end{array}$} & \multicolumn{4}{|c|}{$\begin{array}{l}\text { A.- Objetivo principal: actividad vocacional propiciada } \\
\text { B.- Objetivo secundario } \\
\text { C.- Objetivo potencialmente aplicable } \\
\text { - No se aplica: actividad prohibida o bajo autorización especial o control estricto y regulado }\end{array}$} \\
\hline
\end{tabular}

* Fuente: Adaptado de UICN (1994). Elaboración propia.

Aunque la estrategia proteccionista general de la primera Red Canaria de áreas protegidas creada por la Ley 12/1987, de Declaración de Espacios Naturales Protegidos, tuvo un carácter eminentemente preventivo, su «rediseño» por la Ley 12/1994, de Espacios Naturales de Canarias - y, por ende, la norma legal vigente, esto es, el Texto Refundido de las Leyes de Ordenación del Territorio de Canarias y de Espacios Naturales de Canarias-, se ha dirigido, de manera general, hacia la implementación territorial de estrategias de mitigación, en la medida en que éstas corresponden mayoritariamente a las áreas protegidas 
de la Red Canaria (el 92,5\%), así como a la superficie objeto de protección ambiental (el $97,6 \%$ ), de manera que prácticamente su totalidad es susceptible de acoger un uso turístico de manera adecuada (cuadro 3 ).

Sin embargo, debido a la dificultad de reducir o eliminar completamente la situación de riesgo, resulta que el principal objetivo de ambas estrategias se centra en lograr su calificación como «aceptable». Se trata de un nivel que resulta de la estimación discriminada de la probabilidad de no igualar o sobrepasar un límite de pérdida de calidad ambiental, cuyo valor es considerado, a juicio de las autoridades técnicas que regulan este tipo de decisiones, como lo suficientemente bajo y, por consiguiente, admisible, asumible, tolerable o factible, en la medida que no supera ni la capacidad de asimilación y renovación de los contenidos geográficos objeto de protección ambiental -y, por extensión, de los fundamentos de protección ambiental—, ni los factores «de seguridad» que, en términos deterministas, cubren «razonablemente» la incertidumbre de la posible ocurrencia y severidad de las acciones negativas del uso turístico. El resultado es que las propias conceptuaciones y definiciones jurídicas de las categorías de protección ambiental constituyen una guía para la optimización territorial (física y funcional) del proceso de turistización en las áreas protegidas que comprenden, en la medida en que fijan de manera genérica y teórica lo que podríamos denominar como un «umbral de resistencia del medio al uso turístico» en función del «esquema de tolerancia» de parte o de la totalidad de las mismas. Su amplitud queda definida por dos elementos: por una parte, unos «límites máximos de tolerancia» —o de cambio aceptable- relativos a los usuarios y equipamientos requeridos, y por otro, un «óptimo recreacional», que supone el punto para cuyo valor medio dicho ámbito territorial resulta más eficiente, por cuanto no se produce una saturación y, por tanto, la degradación tanto de su calidad ambiental, como del grado de excelencia de la propia práctica turística.

Dicho esquema determina un comportamiento diferencial en cuanto al nivel adecuado de presión turística, que no sólo se refiere a su presencia/ausencia, sino también a los tipos, comportamientos, ritmos e intensidades de desarrollo, así como a los cánones y reglas de disposición y distribución territorial de sus actividades y equipamientos, en la medida en que condiciona su «potencial turístico» de acuerdo con el grado de vulnerabilidad de los elementos y procesos expuestos del sistema territorial protegido al convertirlo en propicio y concurrente sobre su óptimo - y, por consiguiente, sobre su capacidad de carga turística- según el siguiente procedimiento metodológico: inventario y análisis de los factores de riesgos $\rightarrow$ integración $\rightarrow$ identificación de los factores de riesgos $\rightarrow$ aplicación del criterio de valoración $\rightarrow$ evaluación de riesgos $\rightarrow$ situación de riesgo aceptable (controlado). De esta manera, en consonancia con el planteamiento de que «para que una protección sea eficaz debe ser discriminada, gradual y selectiva, tanto en espacios como en recursos» (Gómez Mendoza, 1995: 408), las categorías de la Red Canaria presentan una capacidad diferencial en cuanto a su uso turístico en función de una jerarquía interna que determina su optimización según un grado de prelación siguiendo la siguiente premisa: mientras que las Reservas Naturales Integrales no son aptas para acogerlo y las Reservas Naturales Especiales lo hacen de manera condicionada, excepcional y puntual espacialmente, el resto de figuras son susceptibles de acogerlo, llegando, incluso, ha concebirse expresamente para ello como sucede con los Parques Nacionales, Parques Naturales, Paisajes Protegidos y Monumentos Naturales, llegando, incluso, a reconocerse formalmente tal vocación en su definición legal; asimismo, mientras que unas categorías no son aptas para un determinado tipo de actividad turística, otras la acogen de manera apropiada sin menoscabo de la calidad ambiental de sus respectivas áreas protegidas. 


\section{Resultados. La definición de los modelos de gestión ambiental del territorio en relación con el uso turístico de las áreas protegidas}

La utilización de las estrategias de manejo de las situaciones de riesgo como el principal criterio de clasificación permite ordenación o agrupar las categorías de protección ambiental - y, por ende, la red completa de áreas protegidas- en función de las formas de gestión territorial, resultado tres tipos de modelos de gestión territorial, que hemos denominado como de preservación, de conservación y mixto; sus características y finalidades se han resumido en el cuadro 2. Éstos son el resultado final del procedimiento de composición de un todo por la reunión de sus partes, de identificación y sistematización de las regularidades y similitudes observadas relativas al tratamiento del uso turístico en la estructura, comportamiento y funcionalidad de las diferentes categorías de protección ambiental, así como la supresión de cualquier detalle incidental siguiendo los principios de la abstracción. Así, como en cualquier modelo, éstos permiten obtener una «imagen conceptual simplificada, cuya virtualidad radica en su capacidad para facilitar la comprensión global del conjunto representado, más que en la fidelidad con que se reprodujo» (Gómez Orea, 2002: 53).

El modelo de preservación supone una estrategia proteccionista preventiva, quedando, por tanto, las categorías sobre las que se aplica exentas de cualquier tipo de uso turístico, debido a su baja - e incluso, nula - capacidad de carga para acoger cualquier tipo de actuación antrópica; en este sentido, responde a una conciencia de que las actividades humanas constituyen agentes «perturbadores» y generadores de cambios no deseados en el sistema territorial protegido. Su efectividad se basa en la aplicación de una serie de medidas de gestión encaminadas a la protección «estricta» de los elementos naturales y procesos ecológicos, para lo cual requiere de la implementación de acciones restrictivas, coercitivas, aislacionistas y estáticas, que suponen una gestión directa y dirigida de las áreas protegidas — territorial proyectado en la norma legislativa de declaración y en el correspondiente proceso y documento de planificación territorial. Implica, por tanto, una «especie de museografía ecológica» (Troitiño, 1995: 33), que, en esencia, responde a un cierto conformismo con la calidad ambiental preoperacional, la cual viene determinada por un predominio prácticamente exclusivo de los componentes abióticos y bióticos. Así, el porcentaje de superficie protegida sobre la que se aplica esta modalidad de gestión supone un indicador del grado de naturalidad del contexto territorial donde se ubican; en este sentido, ésta supone únicamente al 2,4\% de la extensión total de la Red Canaria, la correspondiente a las Reservas Naturales Integrales (cuadro 3).

El modelo de conservación se aplica a las categorías de protección ambiental que presentan una elevada capacidad de carga para acoger diferentes formas de utilización turística, que, en el caso de la Red Canaria, se trata de los Parques Rurales y los Paisajes Protegidos, y, por tanto, a en torno a un tercio de la superficie total de la Red Canaria (cuadro 3). Corresponde a aquéllas donde se produce una presencia — total o parcial— de elementos antrópicos, que, en la mayoría de los casos, constituyen los parámetros de valoración considerados en su declaración como «protegidas». Su efectividad implica -requiere - la continuidad de los usos y aprovechamientos antrópicos, pues su declive o eliminación genera impactos de signo negativo - por subexplotación o pasividad (Gómez Orea, 1992) - , con el consecuente menoscabo de su calidad ambiental, en la medida en que ésta es consecuencia de aquélla, así como un perjuicio para el mantenimiento de los correspondientes fundamentos de protección ambiental. Por tanto, la gestión del riesgo se basa en la implementación de una estrategia de mitigación, la cual se dirige, sobre todo, a 
la reorientación del modelo territorial objeto de protección ambiental, para lo cual, aunque no se excluye la aplicación de medidas de gestión «activa», se fundamenta básicamente en las «pasivas», que suponen una leve acción de control, a modo de mera orientación, de su dinámica territorial (cuadro 2). De esta manera, el proceso de ordenación territorial de las áreas protegidas sobre las que se aplica este modelo, se dirige fundamentalmente a la reconducción de los tipos e intensidades de uso turístico hacia una condición «óptima», con el fin de hacerlos compatibles con las finalidades del régimen de protección ambiental de las categorías de que las comprenden.

Por último, el modelo mixto constituye un «híbrido» de los dos anteriores, de manera que su materialización espacial supone una secuencia en la implementación territorial de medidas propias de los modelos de preservación y conservación; en este sentido, éste es el modelo que predomina mayoritariamente en la Red Canaria, tanto en los relativo al número de áreas protegidas como a la superficie protegida, al corresponder a las figuras de Parque Nacional, Parque Natural, Reserva Natural Especial, Sitio de Interés Científico y Monumento Natural (cuadro 3). Su razón de ser radica en la necesidad de establecer distintos tipos y niveles de uso turístico, medidas y regímenes de ordenación específicos, así como estrategias de gestión de las situaciones de riesgo, en las áreas protegidas de las categorías de protección ambiental sobre las que se aplica, en la medida en que su estructura territorial está configurada por un conjunto de «manchas», a modo de «islotes - teselas- dentro de las islas que constituyen las propias unidades protegidas», y, por tanto, como ámbitos marcadamente heterogéneos en cuanto a su calidad ambiental y capacidad de carga para acoger actividades y equipamientos turísticos. De esta manera, este modelo responde a la multifuncionalidad de las áreas protegidas sobre las que se aplica, la cual se fundamenta en la complementariedad -interrelación e interdependencia- de sus componentes y procesos $\mathrm{y}$, por consiguiente, en la aceptación del binomio «protección ambiental del territorio-desarrollo socioeconómico» con el fin de resolver los conflictos relacionados con la asignación de usos y modos de aprovechamiento turístico. Para ello la aplicación de los tipos de gestión - activa o pasiva - depende de las categorías de protección ambiental (cuadro 2); a este respecto, resulta significativo que las áreas correspondientes a los Sitios de Interés Científico requieren de la implementación de medidas activas únicamente en momentos temporales concretos, en función de las necesidades de protección ambiental de sus contenidos geográficos, como puede ser, por ejemplo, en épocas de reproducción y cría de la fauna o el paso de aves migratorias.

Para ello, la zonificación del ámbito protegido constituye una técnica adecuada para la implementación territorial de estos modelos y, sobre todo, el mixto, en cuanto permite la organización y ordenación interna de las áreas protegidas - $-\mathrm{y}$, por ende, de dicha multifuncionalidad - a través de su sectorización o división en «zonas» en función, para este caso, de las semejanzas en relación con su tolerancia al uso turístico. El resultado final de su correcta ejecución es un «mapa de riesgo», en el que se proyecta adecuadamente la distribución espacial de los efectos potenciales que pueden causar su turistización en función del grado de vulnerabilidad de los elementos y procesos objeto de protección ambiental. Así, siguiendo las determinaciones de la Carta Europea de Turismo Sostenible en Espacios Naturales, la «arquitectura territorial» resultante constituye un mecanismo que canaliza de manera óptima el flujo de usuarios hacia las zonas menos sensibles —más frágiles_- en concreto, aquellas donde se maximice la aptitud del territorio para acoger a las actividades y equipamientos del uso turístico y, a la vez, se minimice el impacto negativo. Por tanto, permite reducir, e incluso, eliminar, las situaciones de conflictividad a favor de las de complementariedad, mediante dos tipos de tácticas de intervención: por un lado, la definición de focos que concentren el uso turístico en zonas concretas, de manera que se elimine la 


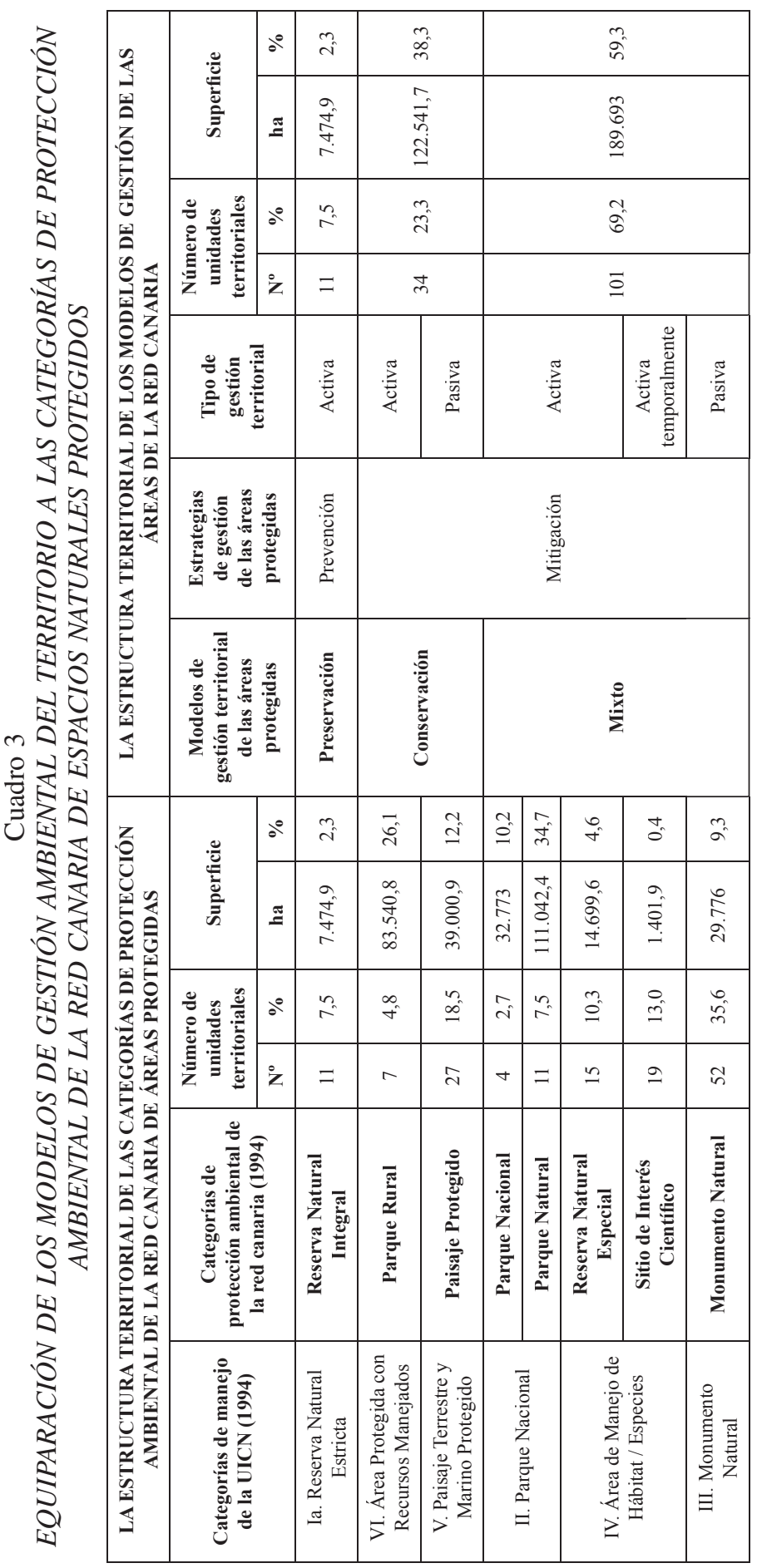

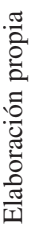


dispersión de los impactos adversos, y por otro, su difusión para evitar la convergencia de los efectos adversos en un único lugar y, por tanto, su saturación.

Consideramos que el modelo de estructura territorial de la zonificación más apropiado es el fundamentado conceptualmente en el de las Reservas de la Biosfera, en la medida en que permite la diferenciación espacial de la aptitud del área protegida para acoger uso turístico de acuerdo a su ausencia/presencia en función a la graduación del riesgo de deterioro o destrucción de su calidad ambiental. El resultado es la organización territorial de las áreas protegidas de la Red Canaria según un esquema espacial configurado por una zona, la denominada en la norma legal como «de exclusión», equiparable a la «zonas núcleo» de las Reservas de la Biosfera, que queda exenta de cualquier tipo de actividad turística, por corresponderse con el ámbito territorial de mayor fragilidad y nula aptitud para acogerla $(\mathrm{R}=0 \mathrm{x} \mathrm{V})$ y en la que se aplica una modalidad de gestión de preservación, rodeada de otras, las «de uso restringido», «moderado» y «general», que, a modo de zonas «tampón o de amortiguación», presentan una desigual capacidad de carga turística: mientras que la primera permite un reducido uso, utilizando medios pedestres y sin que sean admisibles infraestructuras tecnológicas modernas, las otras dos constituyen las más óptimas debido tanto a su menor calidad ambiental relativa dentro del área protegida, como a que admiten una mayor afluencia de usuarios, hasta el punto que, según la propia normativa canaria, las «de uso general» son las más adecuadas para acoger el emplazamiento de las instalaciones, actividades y servicios inherentes al mismo, ajustándose, por tanto, a las modalidades de conservación.

\section{Discusión y conclusiones}

La consideración de las áreas protegidas como recursos de ocio al aire libre genera un flujo de servicios en función de las distintas actividades y equipamientos permitidos, que le otorgan un valor adicional que las hace atractivas y diferentes. Desde esta perspectiva, se ha demostrado que la evaluación de la amenaza que supone su uso turístico es un insumo fundamental para la planificación y ordenación territorial de las áreas protegidas, especialmente cuando se trata de determinar la posible aptitud de la totalidad o de determinadas zonas para acogerlo. Sin embargo, dicha evaluación es sólo una etapa para la determinación del riesgo del proceso de turistización de las áreas protegidas.

Los modelos de gestión territorial de las áreas protegidas definidos constituyen esquemas clasificatorios que, a modo de categoremas y productos de síntesis, constituyen representaciones e interpretaciones conceptuales, simplificadas, selectivas, abstractas, sintéticas, generales e integrales que engloban los rasgos más relevantes y significativos — por su singularidad - del conjunto de elementos - partes - y procesos - interrelaciones - de las estrategias de manejo de las situaciones de riesgo de deterioro de su calidad ambiental por el uso turístico. Así, cada modelo da cabida a las figuras de protección ambiental, de manera que permite la comprensión del conjunto representado y su comparación multiescalar -internacional, nacional y regional_- en cuanto las caracteriza dentro del mismo rango de clasificación independientemente a la propia norma legal que la ha originado $-\mathrm{y}$, por tanto, de las nomenclaturas y definiciones jurídicas sobre la base de las cuales se establecieron tales categorías, aunque su asignación se produjo en función de tal fin-, así como de la consideración de la eficacia del manejo de las áreas protegidas que comprenden, de modo que, en consonancia con las Directrices para las categorías de manejo de áreas protegidas de la UICN (1994), «no constituyen un comentario sobre la misma». Así, conforman mecanismos que permiten «poner orden» entre las partes del todo, integrando a una elevada diversidad de situaciones territoriales objeto de protección ambiental sobre las que se van 
aplicar unos objetivos, comportamientos y, sobre todo, funciones de gestión específicos y homogéneos en relación al uso turístico, los cuales son reflejo de comportamientos diferenciales respecto a la situación de riesgo derivado del mismo, así como a los diversos grados de intervención humana y, por ende, de modificación de la naturalidad del medio. Por consiguiente, el proceso de modelización ha supuesto un ejercicio de normalización de la terminología y la conceptuación de tales figuras, cuyo resultado - los modelos — pretenden funcionar como modalidades universales de gestión ambiental del territorio, por cuanto expresan unas directrices y unos objetivos específicos y semejantes de manejo definidos de acuerdo con su capacidad de carga para acoger uso turístico, el principal objetivo de manejo territorial, así como del esquema previsto de ordenación territorial.

Otra vocación de los modelos de gestión territorial es convertirse en los patrones de asignación de un uso turístico adecuado a las diferentes categorías, en la medida en que su desarrollo óptimo se adecua a la calidad ambiental de sus áreas protegidas, así como a su principal objetivo de gestión territorial, para lo cual fijan los tipos, pautas, formas e intensidades de su desarrollo, así como el conjunto de limitaciones y modos de ordenación territorial dentro y fuera de los ámbitos protegidos. Se revelan, por tanto, como esquemas de optimización para la asignación del uso turístico de las áreas protegidas, en la medida en que posibilitan una estrategia proteccionista que es más eficiente que si se diseña sin conocer el escenario de efectos potenciales del uso turístico de la correspondiente área protegida, dado que permite definir procedimientos de respuesta —optimización— más precisos de acuerdo con el grado de vulnerabilidad de los fundamentos de protección ambiental expuestos. Desde este punto de vista, constituyen en sí mismos «modelos de intervención territorial» de las categorías de protección y, por tanto, de gobierno del territorio de las áreas protegidas que abarcan, por cuanto posibilitan el establecimiento de un principio de compatibilidad entre la actividad antrópica y su calidad ambiental: mientras que el modelo de preservación excluye a las figuras que soportan cualquier modalidad de uso turístico, los de conservación y el mixto fomentan el mantenimiento de ciertos tipos e intensidades, resultando, incluso, el principal objetivo de su gestión.

En definitiva, los modelos de gestión ambiental del territorio contribuyen a la optimización del uso turístico de las áreas protegidas mediante su adecuada planificación y manejo territorial, así como a la implementación del actual corriente del pensamiento proteccionista de que su eficacia y viabilidad no puede fundamentarse en «cercas y multas» (Wearing y Neil, 2000), sino en la integración y compatibilidad de los planteamientos utilitaristas y los dirigidos a la mera salvaguarda de los valores naturales.

\section{Bibliografía}

ANTÓN CLAVÉ, S y VERA REBOLLO, J. F. (1998): «Métodos y técnicas para la planificación turística del territorio», en Oliveras, J. y Antón, S. (eds.): Turismo y planificación del territorio en la España de fin de siglo. Grup d'Estudis Turístics. Universitat Rovira i Virgili. Tarragona, pp. 5-44.

AZCÁRATE Y BANG, T. y ABOAL, J. (1996): Las categorías de protección de los espacios naturales. Un análisis comparado de la legislación española. Viceconsejería de Medio Ambiente del Gobierno de Canarias. Santa Cruz de Tenerife, pp. 53.

BAIGORRI, A. (1999): «De la Naturaleza Social de la Naturaleza», en Pardo, M. (ed.): Sociología Medioambiental. Estado de la cuestión. Fundación de los Ríos. Madrid.

BLANCO PORTILLO, R. y GÓMEZ-LIMÓN GARCÍA, J. (2000): «Gestión del uso público», en EuroparC-españa: Plan de Acción para los Espacios naturales Protegidos del Estado Español. Borrador. Sección del Estado Español de la Federación de Parques Naturales y Nacionales de Europa, 44 pp. 
BUTLER, R.W. (1980): «The concept of a tourist area cycle of evolution: implications for management of resources», en Canadian Geographer, $\mathrm{n}^{\circ}$ 24, pp. 5-15.

CONESA FERNÁNDEZ-VÍTORA, V. (1997): Instrumentos de la gestión ambiental de la empresa. Ediciones Mundi-Prensa. Madrid, $541 \mathrm{pp}$.

CORRAlIZA, J. A.; GARCÍA, J. y VALERO, E. (2002): Los Parques Naturales en España: conservación y disfrute. Fundación Alfonso Martín Escudero. Madrid, pp. 491.

EAGLES, P., McCOOL, S. F. y HAYNES, C. (2002): Sustainable Tourism in Protected Areas: Guidelines for Planning and Management. IUCN. Gland, Switzerland and Cambridge.

FERNÁNDEZ SAÑUDO, P.; PÉREZ CORONA, E. y DE LUCIO, J. V. (1997): Criterios de definición de las categorías de protección de espacios naturales protegidos del Estado Español. Consejería de Medio Ambiente y Desarrollo Regional de la Comunidad de Madrid. Madrid, 28 pp.

GÓMEZ MENDOZA, J. (1995): «Desarrollo rural y espacios naturales protegidos», en Ramos Real, E. y Cruz Villalón, J. (coord.): Hacia un nuevo sistema rural. Ministerio de Agricultura, Pesca y Alimentación. Madrid, pp. 381-410.

GÓMEZ OREA, D. (1992): Evaluación de impacto ambiental. Editorial Agrícola Española. Madrid, $260 \mathrm{pp}$.

GÓMEZ OREA, D. (2002): Ordenación territorial. Ediciones Mundi-Prensa / Editorial Agrícola Española. Madrid, 704 pp.

GÓMEZ-LIMÓN, J.; DE LUCIO FERNÁNDEZ, J. V. y MÚGICA DE LA GUERRA, M. (2000): De la declaración a la gestión activa. Los Espacios Naturales Protegidos del Estado Español en el umbral del siglo XXI. Fundación Fernando González Bernáldez, 94 pp.

HORNBACK, K. y EAGLES, P. (1999): Guidelines for public use measurement and reporting at parks and protected areas. UICN: Gland, Switzerland and Cambridge [www.ahs.uwaterloo. ca/rec/worldww.html]

KAHN, A. E. (1971): The Economics of Regulation: Principles and Institutions. John Wiley \& Son Inc. Nueva York.

LENO CERRO, F. (1993): Técnicas de evaluación del potencial turístico. Ministerio de Industria, Comercio y Turismo. Madrid, $261 \mathrm{pp}$.

LLEÓ DE LA VIÑA, J. (1979): «La actitud prospectiva en la ordenación del territorio», en Revista de Obras Públicas, septiembre 79, pp. 729-730.

MARTÍN ESQUIVEL, J. L. et al (1995): La Red Canaria de Espacios Naturales Protegidos. Viceconsejería de Medio Ambiente. Santa Cruz de Tenerife, $412 \mathrm{pp}$.

MÚGICA, M. y GÓMEZ-LIMÓN, J. (2002): Plan de Acción para los espacios naturales protegidos del Estado Español. Ed. Fundación Fernando González Bernáldez: Madrid, 165 pp.

MÚGICA, M.; GÓMEZ-LIMÓN, J. DE LUCIO, J. y PUERTAS, J. (2004): Anuario EUROPARCEspaña del estado de los espacios naturales protegidos 2003. Ed. Fundación Fernando González Bernáldez. Madrid, $126 \mathrm{pp}$.

MULERO MENDIGORRI, A. (2002): La protección de Espacios Naturales en España. Antecedentes, contrastes territoriales, conflictos y perspectivas. Ediciones Mundi-Prensa. Madrid, 309 pp.

OLCINA CANTOS, J. y AYALA-CARCEDO, F. (2002): «Riesgos naturales. Conceptos fundamentales y clasificación», en AYALA-CARCEDO, F. y OLCINA CANTOS, J. (coord.): Riesgos naturales. Editorial Ariel. Barcelona, pp. 41-73.

O'REILLY, A.M. (1986): «Tourism carrying capacity: concepts and issues», en Tourism Managament, 7 (4): $254-258$.

ORGANISMO AUTÓNOMO PARQUES NACIONALES (2000): Ponencia marco sobre uso público en los Parques Nacionales. Documento interno. 19 pp.

ORGANIZACIÓN MUNDIAL DEL TURISMO (1993): Desarrollo turístico sostenible. Guía para planificadores locales. O.M.T. Madrid.

ORGANIZACIÓN MUNDIAL DEL TURISMO / PROGRAMA DE LAS NACIONES UNIDAS SOBRE MEDIO AMBIENTE (1992): Directrices: ordenación de los parques nacionales y de otras zonas protegidas para el turismo. Serie de Informes Técnicos. Madrid.

PEARCE, P. L. (1993): «Fundamentals of tourist motivation», en Pearce, D.G. y Butler, R.W. (eds.): Tourism Research: Critiques and Challenges. Routledge. Londres. 
RAMOS, A. (dir.) (1979): Planificación física y ecología. Modelos y métodos. EMESA. Madrid, $216 \mathrm{pp}$.

REDONDO ROJAS, C. E. (2000): «Zonificación de los espacios naturales protegidos», en Máster en Ingeniería y Gestión Medioambiental 1999-2000. Escuela de Organización Industrial. 21 pp. [No publicado]

SIMANCAS CRUZ, M. R. (2003): La protección ambiental del territorio y su impacto en las transformaciones recientes de los paisajes rurales de Canarias. Tesis doctoral. Departamento de Geografía. Universidad de La Laguna. 1.188 pp.

SIMANCAS CRUZ, M. R. (2004): «Las áreas protegidas como recurso turístico», en ÁLVAREZ, A.; HERNÁNDEZ, J. y SIMANCAS, M. R. (coordinadores): Turismo y territorio en la sociedad globalizada. Ayuntamiento de Adeje / Instituto Pascual Madoz del Territorio, Urbanismo y Medio Ambiente. Santa Cruz de Tenerife, pp. 217-264.

TROITIÑO VINUESA, M. A. (1995): «Espacios naturales protegidos y desarrollo rural: una relación territorial conflictiva», Boletín de la Asociación de Geógrafos Españoles, nº 20, pp. 7-37.

TROITIÑNO VINUESA, M. A. y otros (2005): «Los espacios protegidos en España: significación e incidencia socioterritorial», en Boletín de la Asociación de Geógrafos Españoles, nº 39, págs. 227-265.

UICN (1994): Guidelines for Protected Area Management Categories. IUCN. Gland, Switzerland and Cambridge.

UNDRO (1979): Natural disasters and vulnerability analysis. Reports of Experts Group Meeting. Geneva.

UNEP-WCMC / UICN (1993): «Directrices para las categorías de manejo de áreas protegidas»[URL: http://www.unepwcmc.org/protected_areas/categories/esp/index.html]

VIÑALS, $M^{\mathrm{a}}$ J. (coord.) (2002): «Guía para la gestión recreativa de los recursos naturales», Viñals, $\mathbf{M}^{\mathrm{a}}$ J. (coord.): Herramientas para la gestión del turismo sostenible en humedales. Serie Cuadernillos Técnicos. Organismo Autónomo Parques Nacionales. Madrid.

WEARING, S. y NEIL, J. (2000): Ecoturismo. Impacto, tendencias y posibilidades. Editorial Síntesis. Madrid, $269 \mathrm{pp}$.

VERA, F.; LÓPEZ PALOMEQUE, F.; MARCHENA, M. y ANTON, S. (1997): Análisis territorial del turismo. Editorial Ariel. Barcelona, 443 pp. 